19

\title{
Влияние пространственной дисперсии в металлах на оптические характеристики биметаллических плазмонных наночастиц
}

\author{
(C) Ю.А. Еремин \\ Московский государственный университет им. М.В. Ломоносова, \\ 119991 Москва, Россия \\ e-mail: eremin@cs.msu.ru
}

Поступила в редакцию 02.02.2021 г.

В окончательной редакции 23.02.2021 г.

Принята к публикации 04.03.2021 г.

\begin{abstract}
Рассмотрена задача дифракции поля электромагнитной плоской волны на слоистой наночастице, составленной из двух плазмонных металлов. На основе метода дискретных источников исследовано влияние пространственной дисперсии в металлах на сечение поглощения. Проведено исследование различных сочетаний металлов ядра и оболочки: $\mathrm{Au} @ \mathrm{Ag}$ и $\mathrm{Ag} @ \mathrm{Au}$ сфероидальных частиц, а также влияния вытянутости и асимметрии геометрии частицы на поглощение энергии. Установлено, что учет пространственной дисперсии как в ядре, так и в оболочке приводит к существенному снижению сечения поглощения и сдвигу плазмонного резонанса в коротковолновую область.
\end{abstract}

Ключевые слова: поверхностный плазмонный резонанс, нелокальный оптический отклик, сфероид.

DOI: $10.21883 /$ OS.2021.08.51205.1872-21

\section{Введение}

Локализованный поверхностный плазмонный резонанс (ПР) представляет собой коллективные колебания электронов проводимости на поверхности раздела плазмонных металлов и диэлектриков при возбуждении падающим светом. Эффект ПР вызывает значительное усиление и локализацию электрического поля вблизи поверхности металла на расстоянии менее длины волны, что позволяет концентрировать электромагнитную энергию в сверхмалых объемах. Это свойство находит широкое практическое применение в солнечной энергетике, устройствах накопления и преобразования энергии, химии, биологии, наномедицине и экологии [1-3].

Наноматериалы ядро-оболочка приобрели в последние годы огромную популярность благодаря их многофункциональным свойствам, которые реализуются посредством манипулирования материалами и размерами ядра и оболочки, а также их геометрией. Продолжающийся прогресс в материаловедении и современные технологии дают возможность синтезировать наноматериалы с заранее заданными физико-химическими свойствами, четко определенными размерами, формой и составом. В результате наноматериалы становятся основой наноплазмоники и наноинженерии [4,5]. Например, эффективность удержания и преобразования солнечного излучения может быть увеличена до 80\% посредством добавления металлических наночастиц в рабочую жидкость солнечных элементов. Этот факт объясняется заметным увеличением фототермической эффективности наночастиц из-за явления ПР [6,7].

Среди слоистых наночастиц биметаллические частицы представляют большой интерес, поскольку их плазмон- ные свойства могут существенно отличаться от свойств составляющих их компонент. Среди различных типов биметаллических наночастиц конфигурации ядро-оболочка были наиболее детально изучены, и было установлено, что связь между ядром и оболочкой может реализовывать ПР со свойствами, которые существенно отличаются от свойств наночастиц, состоящих из каждого отдельного компонента. В химии биметаллические частицы широко используются для улучшения различных процессов преобразования фотоэлектрической энергии, что ведет, например, к повышению эффективности тонкопленочных солнечных элементов (plasmonic nanofluids).

Когда размер частицы или толщина металлического слоя оказывается сравнимым с длиной волны Ферми в этом материале, возникает пространственная нелокальность металла [8]. Для исследования влияния нелокальности (пространственной дисперсии) возможно использовать чисто квантовый подход, основанный на математическом моделировании, в основу которого положено решение уравнения Шредингера, записанного для всей совокупности электронов внутри металла [9]. Однако подобный подход затруднительно применять даже для однородных частиц, размер которых превышает десяток нанометров, особенно в случае рассмотрения металлов с большой плотностью свободных носителей, например золота и серебра. За последнее десятилетие исследователями были разработаны различные подходы, учитывающие возникающие квантовые эффекты, но позволяющие использовать для анализа процессов электромагнитную теорию Максвелла. Одним из таких так называемых квазиклассических подходов, учитывающих пространственную дисперсию, является гидродинамиче- 
ская модель Друде [10] и ее модификации, используемые для описания процессов в слоистых частицах [11]. Эта теория требует учитывать возникновение продольного электрического поля внутри металла, которое отсутствует в классической теории Максвелла. Однако теория Друде нуждается в коррекции некоторых квантовых параметров при рассмотрении несферических плазмонных наночастиц. В качестве альтернативы теории Друде исследовательской группой Мортенсена была разработана теория обобщенного нелокального оптического отклика $(\mathrm{OHO})[12]$. В наших исследованиях мы используем теорию ОНО, которая уже доказала, что является наиболее подходящим инструментом для анализа частиц ядро-оболочка [13]. Теория ОНО позволяет учесть как наличие продольных полей внутри металлов, так и дополнительные граничные условия на поверхностях раздела между различными компонентами. Уже установлено, что подобный подход является адекватным для описания оптических свойств частиц, составленных из благородных металлов, таких как $\mathrm{Ag}$ и $\mathrm{Au}[14]$.

Мы будем использовать теорию ОНО в рамках метода дискретных источников (МДИ) [13,15]. МДИ представляет собой строгий численно-аналитический метод. Он основан на представлении полей в виде линейной комбинации полей мультиполей [13]. В этом случае приближенное решение будет удовлетворять аналитически квазиклассической системе уравнений Максвелла, включающей в себя продольные поля внутри плазмонных металлов. Амплитуды ДИ будут определяются из условий сопряжения на поверхностях раздела сред, включая необходимые для однозначной разрешимости граничной задачи дифракции дополнительные граничные условия. Уникальная особенность МДИ состоит в том, что он позволяет оценить погрешность полученного приближенного решения посредством вычисления невязки полей на поверхностях раздела сред. Все эти обстоятельства уже успешно использовались при исследовании структур ядро-оболочка с учетом пространственной дисперсии при рассмотрении диэлектрических частиц с металлическим покрытием [13,15]. Существенное отличие данной работы от предшествующих заключается в том, что теперь и в ядре и в оболочке присутствуют продольные поля, кроме того, на границе раздела двух плазмонных металлов возникают два дополнительных граничных условий вместо одного, как было раньше.

\section{Постановка граничной задачи дифракции}

Будем полагать, что слоистая биметаллическая частица с осью симметрии $O Z$ целиком располагается в неограниченной области $D_{e}$ пространства. Внутреннее ядро частицы обозначим как $D_{i}$, а область оболочки $D_{s}$. Ограничивающие поверхности ядра и внешнего слоя обозначим как $\partial D_{i, s}$. Будем предполагать, что все среды немагнитные, а их комплексные диэлектрические проницаемости обозначены как $\varepsilon_{v}, v=e, i, s$.

Суть пространственной дисперсии (эффекта нелокальности - ЭН) состоит в том, что связь между смещением $\mathbf{D}(M)$ и полем $\mathbf{E}(M)$ существенно трансформируется, т.е. локальная поточечная связь $\mathbf{D}(M)=\varepsilon(M) \mathbf{E}(M)$ в пространстве заменяется на интегральную $\mathbf{D}(M)=\int\left(M-M^{\prime}\right) \mathbf{E}\left(M^{\prime}\right) d M^{\prime}$ [16]. Следствием ЭН является появление внутри металла продольных электромагнитных полей [17]. Таким образом, поле $\mathbf{E}$ внутри металла перестает быть чисто поперечным $\left(\operatorname{div} \mathbf{E}^{T}=0\right)$, и для адекватного описания происходящих процессов возникает необходимость привлечения дополнительно продольных полей $\left(\operatorname{rot} \mathbf{E}^{L}=0\right)$, которые в случае биметаллической частицы могут возникать как внутри ядра, так и внутри оболочки.

Как уже отмечалось, существенное отличие случая биметаллической частицы от рассмотренного в [13] случая диэлектрической частицы с плазмонным слоем заключается в постановке граничных условий на поверхности $\partial D_{i}$. В работе [15] на поверхности раздела металл-диэлектрик ставилось условие обращения в нуль нормальной компоненты тока проводимости. В данном случае это условие ставится лишь на внешней поверхности $\partial D_{s}$, отделяющей частицу от окружающей среды, в то время как на внутренней поверхности $\partial D_{i}$, разделяющей два плазмонных металла, требуется формулировка совершенно отличных условий сопряжения для полей. В работе [18] были сформулированы общие граничные условия на поверхности, разделяющей два плазмонных материала, которые в общем виде выглядят как

$$
\left[\omega_{p}^{-2}\left(\operatorname{rot} \mathbf{H}-i \omega \varepsilon_{L} \mathbf{E}\right) \cdot \mathbf{n}\right]=0 ; \quad\left[v_{F}^{2} \varepsilon_{L} \operatorname{div} \mathbf{E}\right]=0 .
$$

Здесь скобки [] обозначают скачок величин при переходе через границу раздела, $\omega_{p}$ - плазмонная частота для каждого материала, $v_{F}-$ скорость Ферми в металлах, $\varepsilon_{L}$ соответствует продольному электрическому полю [18]. Подобные условия, адаптированные к конкретному случаю использования сферических функций, уже применялись при рассмотрении слоистых биметаллических сфер [19].

Учитывая уравнения Максвелла и свойства продольного $\mathbf{E}^{L}$ и поперечного $\mathbf{E}^{T}$ полей, легко видеть, что условия (1) могут быть переписаны в следующем виде:

$$
\left[\omega_{p}^{-2}\left(\left(\varepsilon-\varepsilon_{L}\right) \mathbf{E}^{T}-\varepsilon_{L} \mathbf{E}^{L}\right) \cdot \mathbf{n}\right]=0 ; \quad\left[v_{F}^{2} \varepsilon_{L} \operatorname{div} \mathbf{E}^{L}\right]=0 .
$$

Два дополнительных граничных условия на внутренней границе вместо одного, как в [15], обусловлены тем, что внутри металлического ядра теперь также имеется продольное поле.

Запишем теперь постановку граничной задачи дифракции поля плоской линейно поляризованной волны $\left\{\mathbf{E}_{0}, \mathbf{H}_{0}\right\}$, распространяющейся под углом $\pi-\theta$ относительно оси симметрии $O Z$ на слоистой осесимметричной частице (рис. 1). 


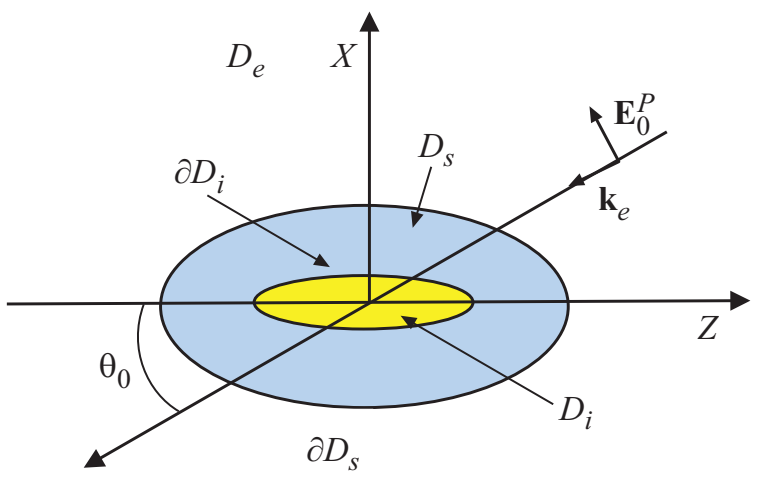

Рис. 1. Слоистая осесимметричная частица.

Граничная задача дифракции с учетом ЭН может быть записана в следующем виде:

$$
\begin{gathered}
\operatorname{rot} \mathbf{H}_{e}=j k \varepsilon_{e} \mathbf{E}_{e} ; \quad \operatorname{rot} \mathbf{E}_{e}=-j k \mathbf{H}_{e} \mathrm{~B} \quad D_{e}, \\
\operatorname{rot} \mathbf{H}_{v}=j k\left(\varepsilon_{v}+\xi_{v}^{2} \text { graddiv }\right) \mathbf{E}_{v}(M) ; \\
\operatorname{rot} \mathbf{E}_{v}=-j k \mathbf{H}_{v} \quad \text { в } \quad D_{v}, \quad v=i, s ; \\
\mathbf{n}_{i} \times\left(\mathbf{E}_{i}(P)-\mathbf{E}_{s}(P)\right)=0, \\
\mathbf{n}_{i} \times\left(\mathbf{H}_{i}(P)-\mathbf{H}_{s}(P)\right)=0, \quad P \in \partial D_{i} ; \\
\left\{\omega_{p}^{-2}\left(\left(\varepsilon-\varepsilon_{L}\right) \mathbf{E}^{T}-\varepsilon_{L} \mathbf{E}^{L}\right)\right\}_{i} \cdot \mathbf{n}_{i} \\
=\left\{\omega_{p}^{-2}\left(\left(\varepsilon-\varepsilon_{L}\right) \mathbf{E}^{T}-\varepsilon_{L} \mathbf{E}^{L}\right)\right\}_{s} \cdot \mathbf{n}_{i} ; \\
\left(v_{F}^{2} \varepsilon_{L} \operatorname{div} \mathbf{E}^{L}\right)_{i}=\left(v_{F}^{2} \varepsilon_{L} \operatorname{div} \mathbf{E}^{L}\right)_{s} \\
\mathbf{n}_{s} \times\left(\mathbf{E}_{s}(P)-\mathbf{E}_{e}(P)\right)=\mathbf{n}_{s} \times \mathbf{E}_{0}(P), \\
\mathbf{n}_{s} \times\left(\mathbf{H}_{s}(P)-\mathbf{H}_{e}(P)\right)=\mathbf{n}_{s} \times \mathbf{H}_{0}(P), \quad P \in \partial D_{s} ; \\
\varepsilon_{L}^{s} \mathbf{n}_{s} \cdot \mathbf{E}_{s}(P)=\varepsilon^{e} \mathbf{n}_{s} \cdot\left(\mathbf{E}_{0}(P)+\mathbf{E}_{0}(P)\right) \\
\lim _{r \rightarrow \infty} r\left(\mathbf{H}_{e} \times \frac{\mathbf{r}}{r}-\sqrt{\varepsilon_{e}} \mathbf{E}_{e}\right)=0, \quad r=|M| \rightarrow \infty .
\end{gathered}
$$

Здесь $\left\{\mathbf{E}_{e}, \mathbf{H}_{e}\right\}$ - рассеянное поле во внешней среде $D_{e},\left\{\mathbf{E}_{i, s}, \mathbf{H}_{i, s}\right\}$ - полные поля в соответствующий областях $D_{i, s}, \mathbf{n}_{i, s}$ - единичные нормали к поверхностям $\partial D_{i, s}, k=\omega / c$, а характеристики среды выбраны таким образом, чтобы $\operatorname{Im} \varepsilon_{e}=0, \operatorname{Im} \varepsilon_{i, s} \leq 0, \operatorname{Im} \varepsilon_{L}^{i, s} \leq 0$. Предполагается, что временная зависимость выбрана в виде $\exp \{i \omega t\}$. Мы будем полагать, что поставленная граничная задача (3) имеет единственное классическое решение.

Квантовые величины $\xi_{i, s}$ и $\varepsilon_{L}^{i, s}$, относящиеся к продольному полю $\mathbf{E}_{i, s}^{L}$, определяются как $\varepsilon_{L}^{v}=\varepsilon_{v}-\omega_{p, v}^{2} / j \gamma_{v}\left(j \gamma_{\nu} \omega-\omega^{2}\right), \quad \xi_{v}^{2}=\varepsilon_{v}\left(\beta_{v}^{2}+D_{v}\left(\gamma_{v}+\right.\right.$ $+j \omega)) /\left(\omega^{2}-j \gamma_{\nu} \omega\right)$. Здесь $\omega_{p, \nu}-$ плазменная частота металла в ядре и оболочке, $\gamma_{v}-$ коэффициенты затухания, $\beta_{v}$ - гидродинамическая скорость в плазме, связанная со скоростями Ферми $v_{F, v}$ соотношением $\beta_{v}^{2}=3 / 5 v_{F, v}^{2}, D_{v}, v=i, s-$ коэффициенты диффузии электронов [16].

\section{Метод дискретных источников}

Перейдем к построению приближенного решения граничной задачи (3), руководствуясь схемой метода дискретных источников для $P / S$-поляризованного излучения [13]. В этом случае поле линейно поляризованной плоской волны для $P$-поляризации имеет вид

$$
\begin{gathered}
\mathbf{E}_{0}^{P}=\left(\mathbf{e}_{x} \cos \theta_{0}+\mathbf{e}_{z} \sin \theta_{0}\right) \psi_{0}(x, z), \\
\mathbf{H}_{0}^{P}=-\sqrt{\varepsilon_{e}} \mathbf{e}_{y} \psi_{0}(x, z),
\end{gathered}
$$

а для $S$-поляризации

$$
\begin{gathered}
\mathbf{E}_{0}^{S}=\mathbf{e}_{y} \psi_{0}(x, z), \\
\mathbf{H}_{0}^{S}=\sqrt{\varepsilon_{e}}\left(\mathbf{e}_{x} \cos \theta_{0}+\mathbf{e}_{z} \sin \theta_{0}\right) \psi_{0}(x, z),
\end{gathered}
$$

где $\quad \psi_{0}(x, z)=\exp \left\{-j k\left(x \sin \theta_{0}-z \cos \theta_{0}\right)\right\}, \quad$ a $\mathbf{e}_{x}, \mathbf{e}_{y}, \mathbf{e}_{z}-$ единичные векторы декартовой системы координат.

Построим приближенное решение задачи (3), которое будет учитывать осевую симметрию рассеивателя и поляризацию внешнего возбуждения [20], удовлетворяя квазиклассической системе уравнений Максвелла во всех областях постоянства параметров среды и условиям излучения на бесконечности. Для построения приближенного решения используются векторные потенциалы следующего вида:

$$
\begin{gathered}
\mathbf{A}_{m n}^{(1) i, e, s \pm}=\left\{Y_{m}^{(1) i, e, s \pm}\left(\eta, z_{n}^{v}\right) \cos (m+1) \varphi ;\right. \\
\left.-Y_{m}^{(1) i, e, s \pm}\left(\eta, z_{n}^{e}\right) \sin (m+1) \varphi ; 0\right\}, \\
\mathbf{A}_{m n}^{(2) i, e, s \pm}=\left\{Y_{m}^{(1) i, e, s \pm}\left(\eta, z_{n}^{v}\right) \sin (m+1) \varphi ;\right. \\
\left.Y_{m}^{(1) i, e, s \pm}\left(\eta, z_{n}^{e}\right) \cos (m+1) \varphi ; \quad 0\right\}, \\
\mathbf{A}_{m n}^{(3) i, e, s \pm}=\left\{0 ; 0 ; Y_{0}^{(1) i, e, s \pm}\left(\eta, z_{n}^{v}\right)\right\}, \quad v=i, e, s .
\end{gathered}
$$

записанные в цилиндрической системе координат. Здесь использованы обозначения $Y_{m}^{i}\left(\eta, z_{n}^{i}\right)=$ $=j_{m}\left(k_{i} r_{\eta z_{n}^{i}}\right) P_{m}^{m}\left(\cos \theta_{z_{n}^{i}}\right), \quad j_{m}() \quad-$.$\quad сферическая$ функция Бесселя, $Y_{m}^{s \pm}\left(\eta, z_{n}^{s}\right)=h_{m}^{(2,1)}\left(k_{s} r_{\eta z_{n}^{s}}\right) P_{m}^{m}\left(\cos \theta_{z_{n}^{s}}\right)$, $h_{m}^{(2,1)}($.$) \quad - сферические функция Ханкеля,$ соответствующие „уходящим“ и „приходящим волнам, $Y_{m}^{e}\left(\eta, z_{n}^{e}\right)=h_{m}^{(2)}\left(k_{e} r_{\eta z_{n}^{e}}\right) P_{m}^{m}\left(\cos \theta_{z_{n}^{e}}\right), P_{m}^{m}\left(\cos \theta_{z_{n}^{v}}\right)=$ $=\left(\rho / r_{\eta z_{n}^{v}}\right)^{m}, r_{\eta z_{n}^{v}}^{2}=\rho^{2}+\left(z-z_{n}^{v}\right), \eta=(\rho, z), k_{v}=k \sqrt{\varepsilon_{v}}$, $z_{n}^{v}$ - положения мультипольных источников на оси вращения, $v=i, e, s$. Заметим, что функции $Y_{m}^{v}\left(\eta, z_{n}^{v}\right) \exp (m+1) \varphi \quad$ удовлетворяют $\quad$ уравнению Гельмгольца.

В свою очередь для случая $P$-поляризации продольное поле строится на основе скалярных потенциалов следующего вида [17]:

$$
\begin{gathered}
\Psi_{m n}^{u, s \pm}(M)=h_{m+1}^{2,1}\left(k_{L}^{v} R_{\eta z_{n}^{v}}\right) P_{m+1}^{m+1}\left(\cos \theta_{z_{n}^{v}}\right) \cos (m+1) \varphi, \\
\Psi_{n}^{i, s \pm}(M)=h_{0}^{2,1}\left(k_{L}^{v} R_{\eta z_{n}^{v}}\right), \quad v=i, s,
\end{gathered}
$$

которые удовлетворяют уравнению Гельмгольца вида $\Delta \Psi_{m n}^{i, s \pm}(M)+\left(k_{L}^{v}\right)^{2} \Psi_{m n}^{i, s \pm}(M)=0$, где $k_{L}^{v}=\sqrt{\varepsilon_{v}} / \xi_{v}$. 
Таким образом, приближенное решение для полей в случае $P$-поляризованного излучения принимает вид

$$
\begin{gathered}
\mathbf{E}_{v}^{T N}=\sum_{m=0}^{M} \sum_{n=1}^{N_{v}^{m}}\left\{p_{m n}^{v} \frac{j}{k \varepsilon_{v}} \operatorname{rot} \operatorname{rot} \mathbf{A}_{m n}^{(1) v}+q_{m n}^{v} \frac{1}{\varepsilon_{v}} \operatorname{rot} \operatorname{rot} \mathbf{A}_{m n}^{(2) v}\right\} \\
+\sum_{n=1}^{N_{v}^{0}} r_{n}^{v} \frac{j}{k \varepsilon_{v}} \operatorname{rot} \operatorname{rot} \mathbf{A}_{n}^{(3) \nu} ; \\
\mathbf{E}_{v}^{T N}=\sum_{m=0}^{M} \sum_{n=1}^{\bar{N}_{\tau}^{m}} \bar{p}_{m n}^{\tau} \operatorname{grad} \Psi_{m n}^{\tau}+\sum_{n=1}^{N_{\tau}} \bar{r}_{n}^{\tau} \operatorname{grad} \Psi_{n}^{\tau} \\
\tau=i, s \pm ; \quad \mathbf{H}_{v}^{N}=\frac{j}{k} \operatorname{rot} \mathbf{E}_{v}^{N}, \quad v=e, i, s \pm
\end{gathered}
$$

Для построения продольного поля в случае S-поляризации используются следующие скалярные потенциалы:

$$
\begin{gathered}
\Psi_{m n}^{i, s \pm}(M)=h_{m+1}^{(2,1)}\left(k_{L}^{v} R_{\eta z_{n}^{v}}\right) P_{m+1}^{m+1}\left(\cos \theta_{z_{n}^{v}}\right) \sin (m+1) \varphi \\
v=i, s
\end{gathered}
$$

а приближенное решение будет иметь вид

$$
\begin{gathered}
\mathbf{E}_{v}^{T N}=\sum_{m=0}^{M} \sum_{n=1}^{N_{v}^{m}}\left\{p_{m n}^{v} \frac{j}{k \varepsilon_{v}} \operatorname{rotrot} \mathbf{A}_{m n}^{(2) v}+q_{m n}^{v} \frac{1}{\varepsilon_{v}} \operatorname{rotrot} \mathbf{A}_{m n}^{(1) v}\right\} \\
+\sum_{n=1}^{N_{v}^{0}} r_{n}^{v} \frac{j}{\varepsilon_{v}} \operatorname{rot} \mathbf{A}_{n}^{(3) v} ; \\
\mathbf{E}_{v}^{T N}=\sum_{m=0}^{M} \sum_{n=1}^{N_{\tau}^{n}} \bar{p}_{m n}^{\tau} \operatorname{grad} \Psi_{m n}^{\tau} ; \quad \tau=i, s \pm \\
\mathbf{H}_{v}^{N}=\frac{j}{k} \operatorname{rot} \mathbf{E}_{v}^{N}, \quad v=e, i, s \pm
\end{gathered}
$$

Заметим, что внутри оболочки $D_{s}$ электромагнитное поле строится как сумма „уходящих“ и „приходящих“ волн, т. е.

$$
\begin{gathered}
\mathbf{E}_{s}^{N}=\mathbf{E}_{s+}^{T N}+\mathbf{E}_{s-}^{T N}+\mathbf{E}_{s+}^{L N}+\mathbf{E}_{s-}^{L N}, \\
\operatorname{div} \mathbf{E}_{s \pm}^{T N}=0, \quad \operatorname{rot} \mathbf{E}_{s \pm}^{L N}=0 .
\end{gathered}
$$

В силу представлений (7) и (8) выражение для $\operatorname{div} \mathbf{E}_{\tau}^{L N}$, $\tau=i, s \pm$, входящее в граничное условие на поверхности $\partial D_{i}$, для $P$-поляризации может быть записано как

$$
\begin{gathered}
\operatorname{div} \mathbf{E}_{\tau}^{L N}=k_{\tau}^{2}\left\{\sum_{m=0}^{M} \sum_{n=1}^{\bar{N}_{\tau}^{m}} \bar{p}_{m n}^{\tau} \Psi_{m n}^{\tau}+\sum_{n=1}^{\bar{N}_{\tau}^{0}} \bar{r}_{n}^{\tau} \Psi_{n}^{\tau}\right\} ; \\
\tau=i, s \pm .
\end{gathered}
$$

и аналогично для $S$-поляризации. Последнее соотношение существенно упрощает условие сопряжения для полей на поверхности $\partial D_{i}$.
Представления для приближенных решений (7) и (8) удовлетворяют всем условиям граничной задачи (3), за исключением условий сопряжения на границах раздела сред. В силу этого неизвестные амплитуды мультиполей $\left\{p_{m n}^{v}, q_{m n}^{v}, r_{n}^{v} ; \bar{p}_{m n}^{\tau}, \bar{r}_{n}^{\tau}\right\}$ определяются из условий сопряжения на поверхностях $\partial D_{i, s}$.

Подробное описание вычислительного алгоритма можно найти в [13]. Отправной его точкой служит разложение плоской волны в ряд Фурье по углу цилиндрической системы координат $\varphi$ :

$$
\begin{aligned}
\exp \left\{-j k_{e} \rho \sin \theta_{0} \cos \varphi\right\}= & \sum_{m=0}^{\infty}\left(2-\delta_{0 m}\right)(-j)^{m} \\
& \times J_{m}\left(k_{e} \rho \sin \theta_{0}\right) \cos m \varphi .
\end{aligned}
$$

Здесь $\delta_{0 m}$ - символ Кронекера, $J_{m}($.$) - цилиндри-$ ческая функция Бесселя. Из условия сходимости ряда определяется максимальное число гармоник Фурье М, участвующее в построении приближенного решения (7) и (8). Поскольку представления для полей (7) и (8) и внешнее возбуждение представлены в виде конечной суммы гармоник Фурье по $\varphi$, это позволяет свести поверхностную аппроксимацию полей к последовательной аппроксимации гармоник Фурье этих полей на образующих поверхностей $\partial D_{i, s}$. Для одномерной аппроксимации используется обобщенный метод коллокаций [21], в рамках которого число точек коллокаций на образующих превышает число мультипольных источников в 2-4 раза. Соответствующие гармоники Фурье представлений для полей (7) и (8) и гармоники падающей плоской волны сшиваются в точках коллокаций. Далее проводится $Q R$-факторизация матриц полученных переопределенных систем для каждой отдельной гармоники и последовательное псевдорешение линейных уравнений одновременно для всего набора углов падения плоской волны $\theta_{0}[22]$. Контроль за погрешностью приближенного решения реализуется посредством вычисления невязки полей в промежуточных точках по отношению к точкам коллокаций. Описанная выше схема вычислительного алгоритма позволяет обеспечивать монотонное уменьшение невязки полей при увеличении числа точек коллокаций и мультипольных источников.

Определив амплитуды ДИ, легко вычислить $\theta, \varphi$-компоненты диаграммы направленности рассеянного поля $\mathbf{F}(\theta, \varphi)$ на единичной сфере $\Omega=\{0 \leq \theta \leq \pi ; 0 \leq \varphi \leq 2 \pi\}$ :

$$
\mathbf{E}_{e}(M) /\left|\mathbf{E}_{0}(M)\right|=\frac{\exp \left\{-j k_{e} r\right\}}{r} \mathbf{F}(\theta, \varphi)+o\left(r^{-1}\right),
$$

$$
r=|M| \rightarrow \infty
$$


Для случая $P$-поляризации они принимают следующий вид:

$$
\begin{gathered}
F_{\theta}^{P}(\theta, \varphi)=j k_{e} \sum_{m=0}^{M}(j \sin \theta)^{m} \cos \{(m+1) \varphi\} \\
\times \sum_{n=1}^{N_{e}^{m}}\left\{p_{m n}^{e} \cos \theta+q_{n m}^{e}\right\} \exp \left\{-j k_{e} z_{n}^{e} \cos \theta\right\} \\
-j k_{e} \sin \theta \sum_{n=1}^{N_{e}^{0}} r_{n}^{e} \exp \left\{-j k_{e} z_{n}^{e} \cos \theta\right\}, \\
F_{\varphi}^{P}(\theta, \varphi)=-j k_{e} \sum_{m=0}^{M}(j \sin \theta)^{m} \sin \{(m+1) \varphi\} \\
\times \sum_{n=1}^{N_{e}^{m}}\left\{p_{m n}^{e}+q_{n m}^{e} \cos \theta\right\} \exp \left\{-j k_{e} z_{n}^{e} \cos \theta\right\} .
\end{gathered}
$$

Совершенно аналогично компоненты диаграммы для случая $S$-поляризации будут

$$
\begin{gathered}
F_{\varphi}^{S}(\theta, \varphi)=j k_{e} \sum_{m=0}^{M}(j \sin \theta)^{m} \sin \{(m+1) \varphi\} \\
\times \sum_{n=1}^{N_{e}^{m}}\left\{p_{m n}^{e} \cos \theta-q_{n m}^{e}\right\} \exp \left\{-j k_{e} z_{n}^{e} \cos \theta\right\}, \\
F_{\theta}^{S}(\theta, \varphi)=j k_{e} \sum_{m=0}^{M}(j \sin \theta)^{m} \cos \{(m+1) \varphi\} \\
\times \sum_{n=1}^{N_{e}^{m}}\left\{p_{m n}^{e} \cos \theta-q_{n m}^{e}\right\} \exp \left\{-j k_{e} z_{n}^{e} \cos \theta\right\} \\
+j k_{e} \sin \theta \sum_{n=1}^{N_{e}^{0}} r_{n}^{e} \exp \left\{-j k_{e} z_{n}^{e} \cos \theta\right\} .
\end{gathered}
$$

Зная компоненты диаграммы рассеяния, легко вычислить все интересующие оптические характеристики в волновой зоне.

\section{Численные результаты}

Нас будет интересовать вычисление сечение рассеяния, которое представляет собой суммарную интенсивность рассеянного поля

$$
\sigma_{\mathrm{scs}}^{P, S}\left(\theta_{0}, \lambda\right)=\int_{\Omega} I^{P, S}\left(\theta_{0}, \theta, \varphi\right) d \Omega .
$$

Здесь интенсивность $I^{P, S}$ на единичной сфере $\Omega=\{0 \leq \theta \leq \pi ; \quad 0 \leq \varphi \leq 2 \pi\} \quad$ определяется соотношением

$$
I^{P, S}\left(\theta_{0}, \theta, \varphi\right)=\left|F_{\theta}^{P, S}\left(\theta_{0}, \theta, \varphi\right)\right|^{2}+\left|F_{\varphi}^{P, S}\left(\theta_{0}, \theta, \varphi\right)\right|^{2} .
$$

Интенсивность рассеяния зависит неявно от угла падения плоской волны $\theta_{0}$, а ее размерность $-\mu \mathrm{m}^{2}$. Кроме того, будем вычислять сечения экстинкции [23], которые применительно к нашему случаю принимают следующий вид:

$$
\begin{gathered}
\sigma_{\text {ext }}^{P}\left(\theta_{0}, \lambda\right)=-\frac{4 \pi}{k_{e}} \operatorname{Im} F_{\theta}^{P}\left(\pi-\theta_{0}, \pi\right) ; \\
\sigma_{\text {ext }}^{S}\left(\theta_{0}, \lambda\right)=\frac{4 \pi}{k_{e}} \operatorname{Im} F_{\varphi}^{S}\left(\pi-\theta_{0}, \pi\right) .
\end{gathered}
$$

Для вычисления сечения поглощения

$$
\sigma_{\mathrm{abs}}\left(\theta_{0}, \lambda\right)=\int_{\partial D_{z}}\left(\mathbf{E}_{e}^{N}+\mathbf{E}_{0}\right) \times\left(\mathbf{H}_{e}^{N}+\mathbf{H}_{0}\right)^{*} d \sigma
$$

мы воспользуемся соотношением вида [23]

$$
\sigma_{\text {ext }}\left(\theta_{0}, \lambda\right)=\sigma_{\text {scs }}\left(\theta_{0}, \lambda\right)+\sigma_{\text {abs }}\left(\theta_{0}, \lambda\right) .
$$

В наших расчетах мы будем рассматривать биметаллические частицы, составленные из золота и серебра. Как уже отмечалось, их термические свойства широко используются в многочисленных практических приложениях $[24,25]$. Мы начнем наше рассмотрение с частиц сферической формы и оценим влияние ЭН на положение и амплитуду плазмонного резонанса, следуя работе [26]. Отметим, что учет частотной дисперсии показателей преломления $\mathrm{Au}$ и $\mathrm{Ag}$ осуществлялся на основе показателей преломления, взятых из базы данных [27].

Для вычисления нелокальных величин $\varepsilon_{L}$ и $k_{L}$ с целью анализа влияния ЭН на сечения (14) и (15) понадобятся соответствующие квантовые параметры, которые для золота Аu выбраны равными

$$
\begin{gathered}
\hbar \omega_{p}=9.02 \mathrm{eV}, \quad \hbar \gamma=0.071 \mathrm{eV}, \\
\nu_{F}=1.39 \cdot 10^{12} \mu \mathrm{m} / \mathrm{s}, \quad D=8.62 \cdot 10^{6} \mu \mathrm{m}^{2} / \mathrm{s},
\end{gathered}
$$

a для серебра $\mathrm{Ag}$

$$
\begin{gathered}
\hbar \omega_{p}=8.99 \mathrm{eV}, \quad \hbar \gamma=0.025 \mathrm{eV}, \\
v_{F}=1.39 \cdot 10^{12} \mu \mathrm{m} / \mathrm{s}, \quad D=9.62 \cdot 10^{6} \mu \mathrm{m}^{2} / \mathrm{s} .
\end{gathered}
$$

Эти значения взяты из работы [16]. Задавая длину волны внешнего возбуждения $\lambda$, вычисляя соответствующее значение частоты $\omega$, легко определить значения нелокальных параметров $\varepsilon_{L}$ и $k_{L}$, участвующих в расчетах.

В работе [26] был рассмотрен локальный случай биметаллических слоистых $\mathrm{Ag} @ \mathrm{Au}(\mathrm{Ag}$ - ядро, $\mathrm{Au}-$ оболочка) и $\mathrm{Au} @ \mathrm{Ag}$ частиц сферической формы, внешний диаметр которых выбирался равным $D_{e}=20 \mathrm{~nm}$, а внутренний диаметр изменялся от 0 до $20 \mathrm{~nm}$ посредством варьирования толщины оболочки $d$. В качестве иллюстрации влияния ЭН для этого случая мы выбрали результаты, изображенные на рис. 5, $a, b$ статьи [26]. Это сечения экстинкции $\sigma_{\text {ext }}$ для слоистых частиц $\mathrm{Ag} @ \mathrm{Au}$ и $\mathrm{Au} @ \mathrm{Ag}$ с фиксированным внешним 


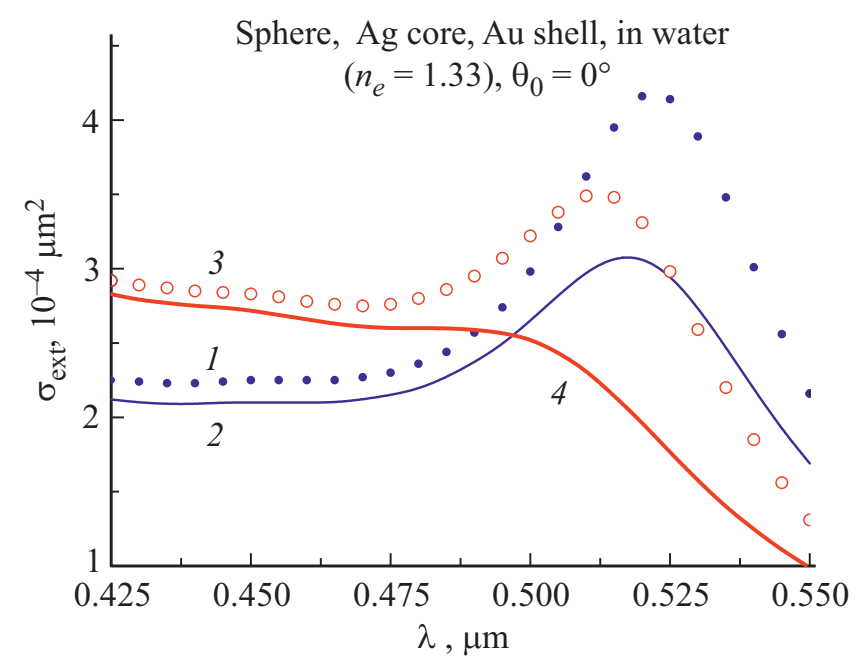

Рис. 2. Спектральная зависимость сечения экстинкции (15) сферической частицы $\mathrm{Ag} @ \mathrm{Au}$ с ядром диаметром $D=6 \mathrm{~nm} \quad(1,2)$ или $12 \mathrm{~nm} \quad(3,4)$ и оболочкой толщиной $d=7 \mathrm{~nm}(1,2)$ или $4 \mathrm{~nm}(3,4)$ в воде с показателем преломления ne $=1.33$ при падении волны под углом 0 . Локальный $(1,3)$ и нелокальный $(2,4)$ случаи.

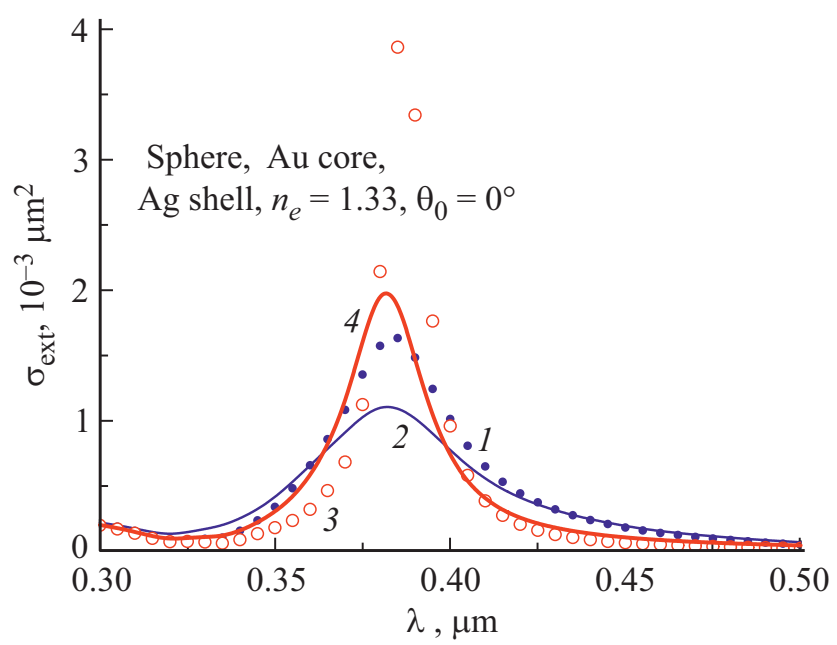

Рис. 3. Спектральная зависимость сечения экстинкции (15) сферической частицы $\mathrm{Au} @ \mathrm{Ag}$ с ядром диаметром $D=10 \mathrm{~nm}(1,2)$ или $6 \mathrm{~nm}(3,4)$ и оболочкой толщиной $d=5 \mathrm{~nm}(1,2)$ или $7 \mathrm{~nm}(3,4)$, при падении волны под углом $0^{\circ}, n_{e}=1.33$. Локальный $(1,3)$ и нелокальный $(2,4)$ случаи.

диаметром $D=20 \mathrm{~nm}$ и различной толщиной пленки $d$, расположенных в воде $n_{e}=1.33$. Полученные численные результаты можно видеть на рис. 2 и 3.

Из рассмотрения данных результатов можно сделать сразу несколько важных выводов:

1) учет ЭН приводит к существенному, до $50 \%$, снижению величины ПР;

2) для частицы $\mathrm{Ag} @ \mathrm{Au}$ ПР располагается вблизи $520 \mathrm{~nm}$, в то время как для $\mathrm{Au} @ \mathrm{Ag}$ он почти на порядок больше, но сдвинут в область более коротких волн, к $380 \mathrm{~nm}$.
Биметаллические частицы применяются в тонкопленочных элементах солнечных батарей, где их использование позволяет повысить эффективность преобразования солнечного света до 80\% [7]. Поскольку в этом случае основную роль играют фототермические свойства биметаллических частиц, то мы будем теперь интересоваться сечением поглощения (14) частиц, помещенных в этиленгликоль $\left(n_{e}=1.432\right)$, который служит наполнителем подобных элементов [7]. Кроме того, поскольку наиболее интересным диапазоном в случае с солнечными элементами является диапазон длин волн от 450 до $550 \mathrm{~nm}$, то мы будем стремиться одновременно попасть в этот диапазон и увеличить амплитуду ПР. Для этого, следуя предыдущему опыту [13], исследуем влияние вытянутости сфероидальной частицы на положение и амплитуду ПР. Отметим, что использование слоистых частиц сфероидальной формы на практике не является уникальным [28].

На рис. 4 и 5 изображены результаты, демонстрирующие влияние вытянутости сфероида $r$ на положение и амплитуду ПР для $\mathrm{Ag} @ \mathrm{Au}$ и $\mathrm{Au} @ \mathrm{Ag}$ частиц с эквиобъемным внутренним диаметром $D=6 \mathrm{~nm}$ и толщиной оболочки $d=7 \mathrm{~nm}$. Легко видеть, что увеличение вытянутости ведет одновременно к смещению вправо и увеличению амплитуды ПР. При этом амплитуда ПР для $\mathrm{Ag} @ \mathrm{Au}$ существенно отстает от амплитуды для частиц $\mathrm{Au} @ \mathrm{Ag}$. Кроме того, во всех случаях учет влияния ЭН приводит к существенному снижению сечения поглощения в положении резонанса при небольшом сдвиге в область коротких волн.

Перейдем к анализу влияния асимметрии толщины внешней оболочки для случая $\mathrm{Au} @ \mathrm{Ag}, D=6 \mathrm{~nm}$, $d=5 \mathrm{~nm}$. Результаты для сферического случая представ-

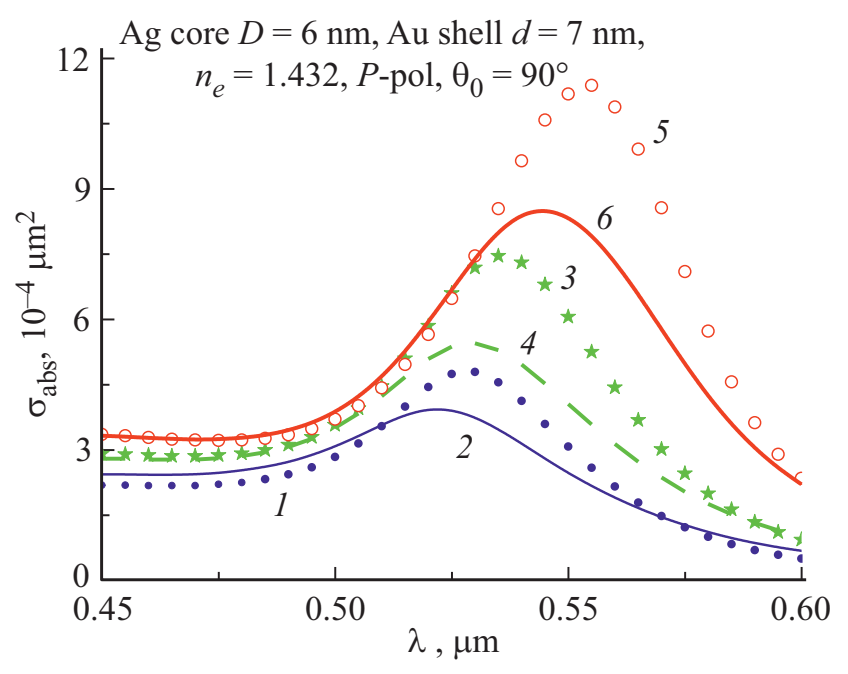

Рис. 4. Спектр поглощения (зависимость сечения поглощения от длины волны) (14) сферической $(1,2)$ и сфероидальной с $r=1.5(3,4)$ или $2.5(5,6)$ частиц $\mathrm{Ag} @ \mathrm{Au}$ с ядром диаметром $D=6 \mathrm{~nm}$ и оболочкой толщиной $d=7 \mathrm{~nm}$, при падении волны под углом $90^{\circ}, n_{e}=1.432$. Локальный $(1,3,5)$ и нелокальный $(2,4,6)$ случаи. 


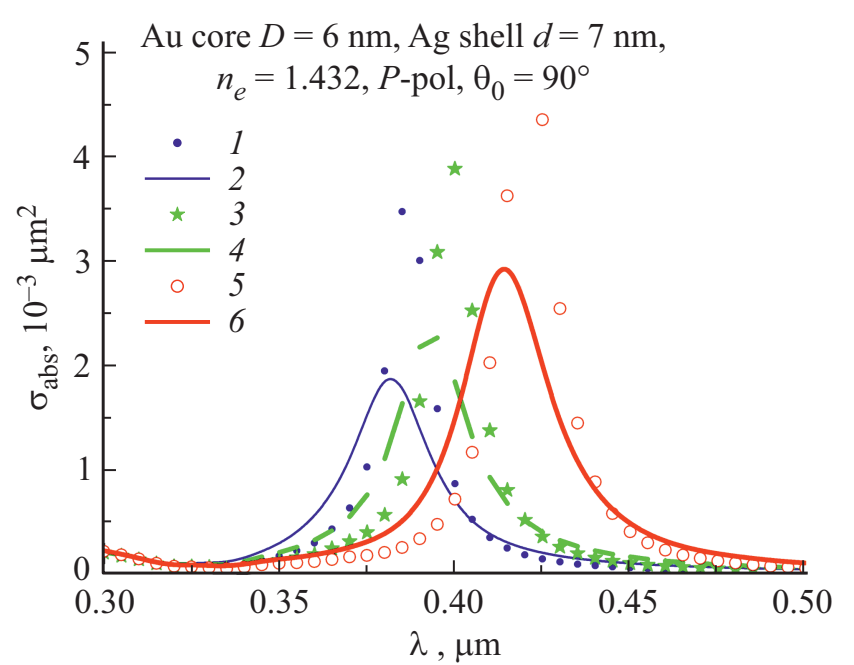

Рис. 5. Спектр поглощения (14) сферической $(1,2)$ и сфероидальной с $r=1.5(3,4)$ или $r=2.5(5,6)$ частиц $\mathrm{Au} @ \mathrm{Ag}$ с ядром диаметром $D=6 \mathrm{~nm}$ и оболочкой толщиной $d=7 \mathrm{~nm}$ при падении волны под углом $90^{\circ}, n_{e}=1.432$. Локальный $(1,3,5)$ и нелокальный $(2,4,6)$ случаи.

Sphere, Au core $D=6 \mathrm{~nm}, \mathrm{Ag}$ shell $d=7 \mathrm{~nm}$,

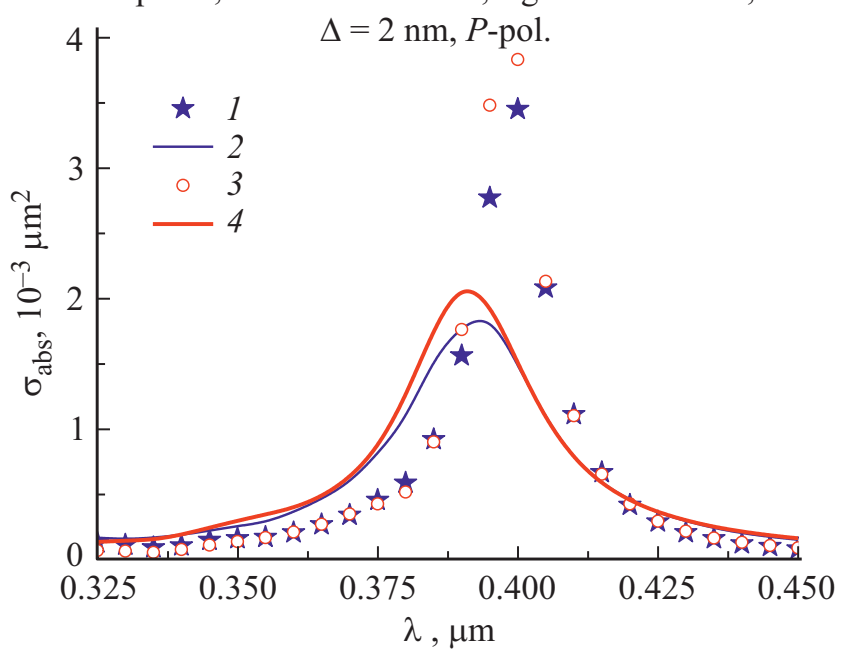

Рис. 6. Спектр поглощения сферической частицы $\mathrm{Au} @ \mathrm{Ag} \mathrm{c}$ $D=6 \mathrm{~nm}$ и $d=7 \mathrm{~nm}$, смещение $\Delta=2 \mathrm{~nm} . \theta_{0}=0(1,2)$ или $90^{\circ}(3,4)$, локальный $(1,3)$ и нелокальный $(2,4)$ случаи.

лены на рис. 6. При этом сдвиг центра внутренней сферы по отношению к внешней составляет $2 \mathrm{~nm}$, тем самым толщина внешней оболочки меняется от 9 до $5 \mathrm{~nm}$. Результаты для сфероида с соотношением осей изображены на рис. 7. Из результатов видно, что такой сдвиг практически не приводит к заметному изменению амплитуды ПР или его расположения.

Другим фактором, который позволяет осуществлять сдвиг ПР в длинноволновую область, является уменьшение толщины внешнего слоя [29], оставляя внешний эквиобъемный диаметр постоянным.
Результаты для сфероида с соотношением осей $r=2$ и различными толщинами внешней оболочки можно видеть на рис. 8. Действительно при толщине внешнего слоя $d=5 \mathrm{~nm}$ максимум сечения поглощения располагается в нужном диапазоне. Однако следует заметить, что частицы в слое могут располагаться хаотически по отношению к направлению внешнего излучения. В этом плане представляется уместным сравнить поведение $P$ и $S$-поляризаций. Как явствует из рис. 9, максимум для $S$-поляризации существенно меньше по амплитуде и сдвинут влево по отношению к максимуму для $P$-поляризации. Это закономерно, так как при угле падения $90^{\circ}$ вектор $\mathbf{E}_{0}$ для $P$-поляризации параллелен

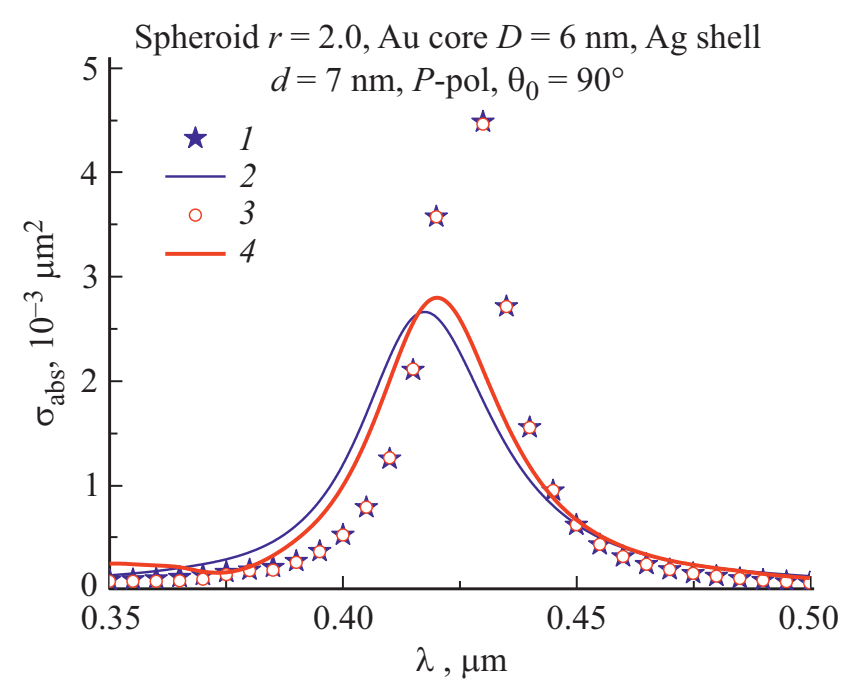

Рис. 7. Спектр поглощения сфероида $\mathrm{Au} @ \mathrm{Ag}$ с $r=2.0$, $D=6 \mathrm{~nm}, d=7 \mathrm{~nm}, \Delta=2 \mathrm{~nm} . \theta_{0}=0(1,2)$ или $90^{\circ}(3,4)$, локальный $(1,3)$ и нелокальный $(2,4)$ случаи.

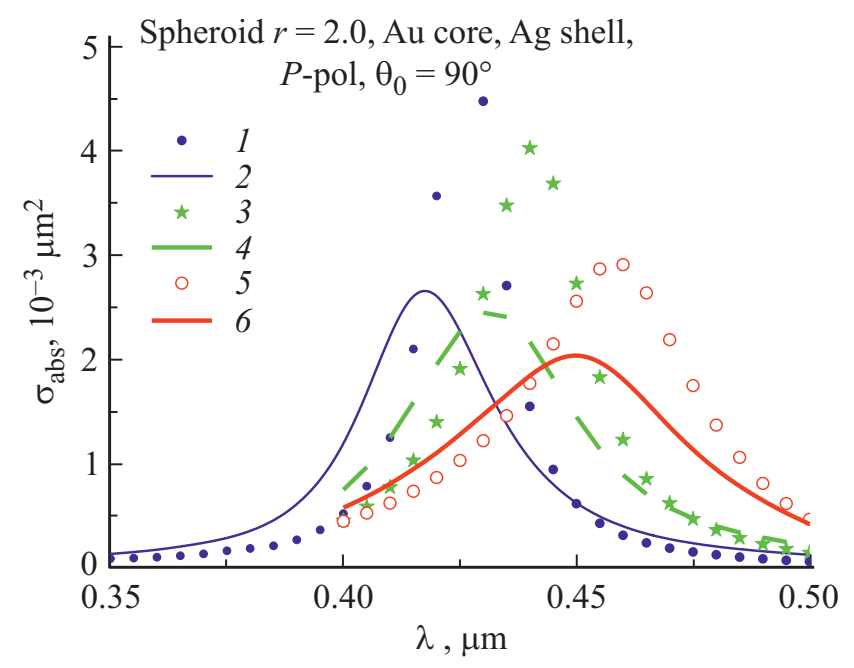

Рис. 8. Спектр поглощения сфероида $\mathrm{Au} @ \mathrm{Ag}$ с $r=2.0$, $D=6 \mathrm{~nm}$ и $d=7 \mathrm{~nm}(1,2), D=8 \mathrm{~nm}$ и $d=6 \mathrm{~nm}(3,4)$, $D=10 \mathrm{~nm}$ и $d=5 \mathrm{~nm}(5,6)$ при падении света под углом $90^{\circ}$, Р-поляризация, локальный $(1,3,5)$ и нелокальный $(2,4,6)$ случаи. 


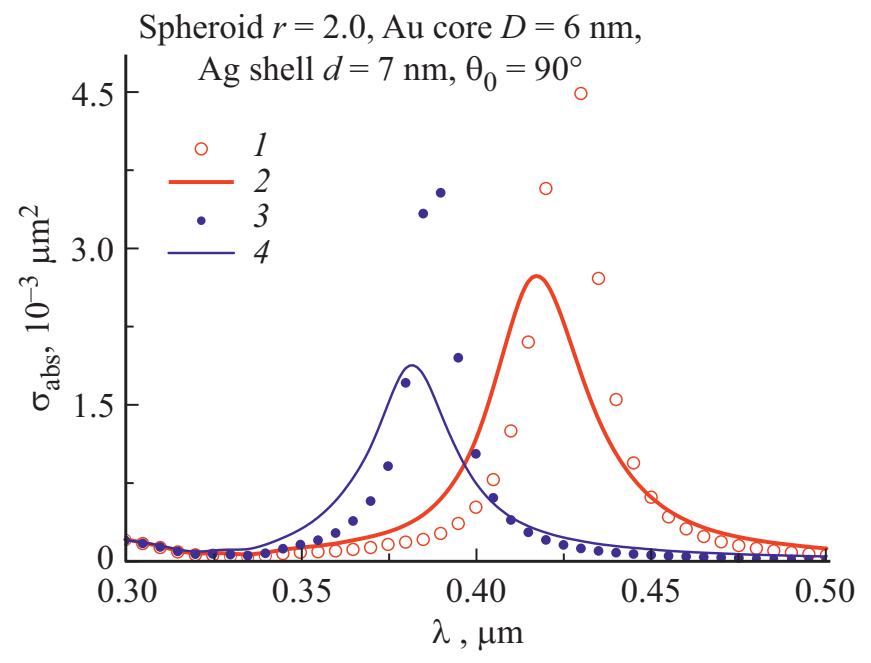

Рис. 9. Спектр поглощения сфероида $\mathrm{Au} @ \mathrm{Ag}$ с $r=2.0$, $D=6 \mathrm{~nm}, d=7 \mathrm{~nm}$, при падении света под углом 90.$P$ - $(1,2)$ и $S$ - $(3,4)$ поляризация, локальный $(1,3)$ и нелокальный $(2,4)$ случаи.

большей оси сфероида, в то время как для $S$-поляризации он ей перпендикулярен.

\section{Заключение}

Резюмируя проведенные исследования остановимся на нескольких основных выводах.

1. Метод дискретных источников был успешно адаптирован применительно к анализу оптических характеристик биметаллических частиц с учетом пространственной дисперсии.

2. В результате численного эксперимента установлено, что слоистые частицы $\mathrm{Au} @ \mathrm{Ag}$ реализуют большие значения сечения поглощения, но длина волны его максимума короче $450 \mathrm{~nm}$.

3. Частицы Ag@Au имеют меньшее сечение поглощения, но его максимум попадает в диапазон длин волн более $500 \mathrm{~nm}$.

4. Учет пространственной дисперсии оказывает весьма заметное влияние и ведет к существенному снижению величины сечения поглощения до $40-50 \%$ и сдвигу максимума в область коротких волн на 5-10 nm.

\section{Финансирование работы}

Работа выполнена при финансовой поддержке Российского фонда фундаментальных исследований (проект № 20-01-00558).

\section{Конфликт интересов}

Автор заявляет, что у него нет конфликта интересов.

\section{Конфликт интересов}

Авторы заявляют, что у них нет конфликта интересов.

\section{Список литературы}

[1] Pelton M., Bryant G. Introduction to Metal-Nanoparticle Plasmonics. John Wiley \& Sons, 2013. 296 p.

[2] Chon J.W.M., Iniewski K. Nanoplasmonics. Advanced Device Applications. CRC Press. 2018. 288 p. doi 10.1201/9781315216423

[3] Stockman M.I., Kneipp K., Bozhevolnyi S.I. et al. // J. Opt. 2018. V. 20. P. 043001. doi 10.1088/2040-8986/aaa114

[4] Feng H.-P., Tang L., Zeng G.-M., Zhou Y. et al. // Adv. Colloid Interface Sci. 2019. V. 267. P. 26. doi.org/10.1016/j.cis.2019.03.001

[5] Kalambate P.K., Dhanjai., Huang Z., Li Y. et al. // Trends Anal. Chem. 2019. V. 115. P. 147. doi 10.1016/j.trac.2019.04.002

[6] Chen M., He Y., Wang X., Hu Y. // Appl. Energy. 2018. V. 211. P. 735. doi 10.1016/j.apenergy.2017.11.087

[7] Farooq S., Vitalb C.V.P., Gymez-Malagyn L.A., de Araujo R.E., Rativa D. // Solar Energy. 2020. V. 208. P. 1181. doi 10.1016/j.solener.2020.08.068

[8] Xu D., Xiong X., Wu L., Ren X-F. et al. // Adv. Opt. Photonics. 2018. V. 10. N 4. P. 703. doi 10.1364/AOP.10.000703

[9] Barbry M., Koval P., Marchesin F., Esteban R. et al. // Nano Lett. 2015. V. 15. P. 3410. doi 10.1021/acs.nanolett.5b00759

[10] Ciraci C., Pendry J.B., Smith D.R. // Chem. Phys. Chem. 2013. V. 14. P. 1109. doi 10.1002/cphc.201200992

[11] Khalid M., Cirací C. // Photonics. 2019. V. 6. N 2. P. 39. doi 10.3390/photonics6020039

[12] Tserkezis C., Yan W., Hsieh W., Sun G. et al. // Int. J. Mod. Phys. B. 2017. V. 31. P. 1740005. doi $10.1142 / \mathrm{S} 0217979217400057$

[13] Eremin Yu., Doicu A., Wriedt T. // J. Opt. Soc. Am. A. 2020. V. 37. N 7. P. 1135 . doi 10.1364/JOSAA. 392537

[14] Kupresak M., Zheng X., Vandenbosch G.A.E., Moshchalkov V.V. // Adv. Theory Simul. 2019. V. 3. P. 1900172. doi 10.1002/adts.201900172

[15] Еремин Ю.А. // Опт. спектр. 2020. Т. 128. № 9. С. 1388. doi 10.21883/OS.2020.09.49881.141-20;

Eremin Yu.A. // Opt. Spectrosc. 2020. V. 128. N 9. P. 1500. doi 10.1134/S0030400X20090088

[16] Wubs M., Mortensen A. // Quantum Plasmonics. / Ed. by Bozhevolnyi S.I. et al., Springer, Switzerland. 2017. P. 279-302. doi 10.1007/978-3-319-45820-5_12

[17] Лифииц Е.М., Питаевский Л.П. Физическая кинетика. М.: Наука, 1978. С. 167.

[18] Boardman A.D., Ruppin R. // Surface Sci. 1981. V. 112. N 1-2. P. 153. doi 10.1016/0039-6028(81)90339-3

[19] Dong T., Shi Y., Liu H. et al. // J. Phys. D: Appl. Phys. 2017. V. 50. P. 495302. doi 10.1088/1361-6463/aa9257

[20] Гришина Н.В., Еремин Ю.А., Свешников А.Г. // Опт. спектр. 2012. Т. 113. № 4. С. 440; Grishina N.V., Eremin Yu.A., Sveshnikov A.G. // Opt. Spectr. 2012. V. 113. N 4. P. 440. doi 10.1134/S0030400X12100049

[21] Бахвалов Н.С. Численные методы. М.: Наука, 1975. 632 с.

[22] Воеводин В.В., Кузнецов Ю.А. Матрицы и вычисления. М.: Наука, 1984. 320 c. 
[23] Newton R.G. Scattering Theory of Waves and Particles. McGraw Hill, 1966. 746 c.

[24] Zhu J., Li X., Li J.-J., Zhao J-W. // Spectrochimica Acta. Part A: Molecul. Biomolecul. Spectr. 2018. V. 189. P. 571. doi 10.1016/j.saa.2017.08.064

[25] Radhu S. // Materials Today. 2020. V. 25. Part 2. P. 285. doi 10.1016/j.matpr.2020.01.413

[26] Zhang Ch., Chen B.-Q., Li Z.-Y. et al. // J. Phys. Chem. C. 2015. V. 119. N 29. P. 16836. doi 10.1021/acs.jpcc.5b04232

[27] Johnson P.B., Christy R.W. // Phys. Rev. B. 1972. V. 6. P. 4370. doi 10.1103/PhysRevB.6.4370

[28] Bhatia P., Verma S.S., Sinha M.M. // Phys. Lett. A. 2019. V. 383. P. 2542. doi 10.1016/j.physleta.2019.05.009

[29] Eremina E., Eremin Y., Wriedt T. // J. Modern Opt. 2008. V. 55. N 2. P. 297. doi $10.1080 / 09500340701433332$ 